\title{
p53 shades of Hippo
}

\author{
Noa Furth ${ }^{1}$, Yael Aylon ${ }^{\star, 1}$ and Moshe Oren ${ }^{\star, 1}$
}

The three p53 family members, p53, p63 and p73, are structurally similar and share many biochemical activities. Yet, along with their common fundamental role in protecting genomic fidelity, each has acquired distinct functions related to diverse cell autonomous and non-autonomous processes. Similar to the p53 family, the Hippo signaling pathway impacts a multitude of cellular processes, spanning from cell cycle and metabolism to development and tumor suppression. The core Hippo module consists of the tumor-suppressive MST-LATS kinases and oncogenic transcriptional co-effectors YAP and TAZ. A wealth of accumulated data suggests a complex and delicate regulatory network connecting the p53 and Hippo pathways, in a highly context-specific manner. This generates multiple layers of interaction, ranging from interdependent and collaborative signaling to apparent antagonistic activity. Furthermore, genetic and epigenetic alterations can disrupt this homeostatic network, paving the way to genomic instability and cancer. This strengthens the need to better understand the nuances that control the molecular function of each component and the cross-talk between the different components. Here, we review interactions between the p53 and Hippo pathways within a subset of physiological contexts, focusing on normal stem cells and development, as well as regulation of apoptosis, senescence and metabolism in transformed cells.

Cell Death and Differentiation (2018) 25, 81-92; doi:10.1038/cdd.2017.163; published online 6 October 2017

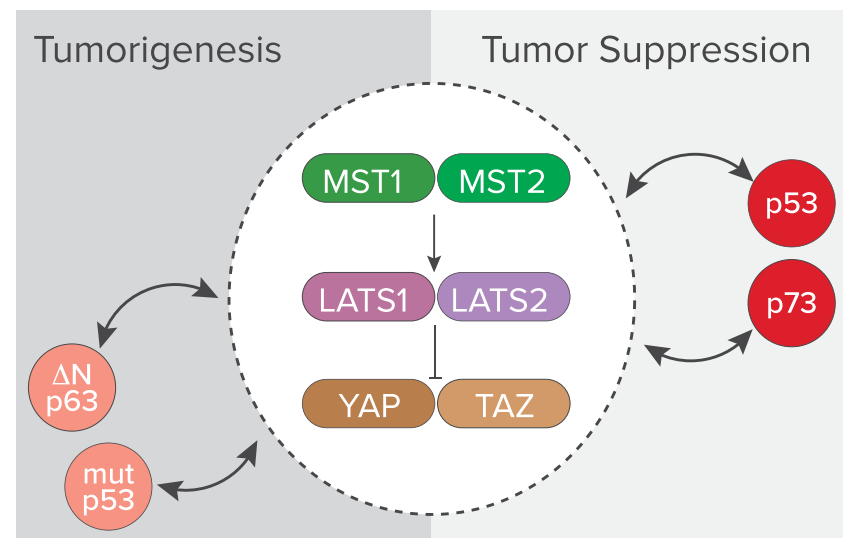

Graphical Abstract

p53 family members and the core components of the Hippo pathway interact on multiple levels. Together, they comprise a sensitive and context-dependent signaling network that exerts a profound and diverse impact on cell fate. Within the context of tumor development, the core components of the Hippo pathway cooperate with p53 and p73 to suppress tumorigenesis, whereas mutant forms of $\mathrm{p} 53$ and $\Delta \mathrm{Np} 63$ can cooperate with the Hippo effectors YAP and TAZ to promote tumorigenesis.

\section{Facts}

- The balance between self-renewal and differentiation in embryonic and somatic stem cells involves multi-layered interactions between p53, p63, LATS2 and YAP.

- The tumor-suppressive functions of the Hippo pathway kinases and the pro-apoptotic functions of YAP are often mediated by p53 or p73.

- Oncogenic RAS signaling is a prominent activator of the Hippo-p53 signaling network.
- Maintaining metabolic homeostasis requires signaling between LATS2 and p53, whereas rewiring of cancer cell metabolism engages the oncogenic functions of both mutant p53 and YAP.

\section{Open Questions}

- What are the upstream cues that sway YAP function from promoting TEAD-dependent proliferation toward p73/p53dependent apoptosis?

- How dynamic are the functional states of p53, and to what extent does the Hippo pathway modulate tumorsuppressive versus pro-survival functions of wild-type p53?

- What determines whether mutant p53 functionally interacts with YAP or TAZ, and how does this interaction change mutant p53-dependent phenotypes?

In approximately half of human cancers TP53 is mutated; in many of the remaining half, the function of the retained wild-type (wt) p53 protein is compromised by deregulation of upstream or downstream regulators. ${ }^{1}$ Functionally, p53 inhibits the proliferation of potentially tumorigenic cells, chiefly through transcriptionally instigating cell cycle arrest, differentiation, senescence or apoptosis. ${ }^{2}$ Cancer-associated mutations in p53 may directly disrupt p53-DNA interactions or drive conformational changes in the p53 protein, in both cases leading to loss of transcriptional activity and tumor-suppressor capabilities. ${ }^{3}$ Mutations in the TP53 gene may also convey an additional selective advantage for tumors, as the mutant p53 (mutp53) protein may acquire cancer-promoting activities, augmenting cell migration, invasion and tumorigenesis. ${ }^{3}$ This

\footnotetext{
1Department of Molecular Cell Biology, The Weizmann Institute, Rehovot, Israel ${ }^{*}$ Corresponding author: Y Aylon or M Oren, Department of Molecular Cell Biology, The Weizmann Institute, POB 26, 234 Herzl Street, Rehovot 7610001, Israel. Tel: +972 89342358; Fax: +972 89346004; E-mail: yael.aylon@weizmann.ac.il or moshe.oren@weizmann.ac.il

Received 16.5.17; revised 15.8.17; accepted 30.8.17; Edited by F Pentimalli; published online 06.10.17
} 
mutp53 gain-of-function (GOF) is executed often by 'piggybacking' on other transcription factors or signaling pathways and enables the mutp53 to deregulate metabolic pathways, increase metastasis and enhance chemotherapy resistance. $^{4-8}$ Recently, pro-survival functions have been attributed also to wt p53, with some activities shared between the wt and mutant isoforms. ${ }^{3,9-13}$ This may reflect a physiological role for p53 in maintaining homeostasis in the face of transient or fluctuating stress, and may confer contextdependent selective advantages also in those tumors that retain wt $\mathrm{p} 53 .^{10}$

The p53 family of transcription factors consists of three paralogs, p53, p63 and p73, which evolved from a common ancestor through two gene duplication events. ${ }^{14}$ Interestingly, in organisms, which possess a single p53 family gene, for example, C. Elegans and Drosophila, this gene is more similar to an ancestral p63/p73 hybrid gene and less to p53 itself. $^{14}$ Duplication events enabled the family members to expand their function and engage in additional cellular processes and signaling pathways, while retaining some common activities. ${ }^{14}$ Similar to p53, p63 and p73 are nuclear proteins that bind to canonical p53 DNA-binding sites and can transactivate p53responsive promoters. Both are principally recognized for their role in development, with p63 necessary for skin development and p73 linked to the formation of the nervous system. ${ }^{15-17}$ On top of their developmental roles, recent studies have determined that p63 and p73 are critical for the maintenance of genomic integrity and response to DNA damage in adult organisms. ${ }^{18,19}$ Like TP53, also the $p 63$ and $p 73$ genes can be transcribed from two alternative promoters and are alternatively spliced, ${ }^{20}$ giving rise to a profusion of isoforms. ${ }^{21-23}$ Of relevance to this review, the p63 and p73 isoforms can be roughly categorized into two groups: transactivation (TA) domain isoforms, which structurally resemble full-length p53 and act as tumor suppressors, and the $\Delta \mathrm{N}$ isoforms, which in some cases can bind to p53, TAp63 or TAp73 to inhibit their function and promote tumorigenesis. ${ }^{24}$

Differential upstream signaling distinctly impacts p53 family members' stability and activity. Upon DNA damage, p53 is phosphorylated by numerous kinases, whereas p73 is primarily phosphorylated by c-Abl. ${ }^{25-27}$ Similarly, MDM2 is the primary E3 ubiquitin ligase for p53 but not p73, ${ }^{28,29}$ whereas ITCH has been shown to control the stability of both p63 and p73 but does not promote the degradation of p53. ${ }^{30,31}$ Another protein that differentially binds members of the p53 family is YAP. YAP binds p63, p73 and mutp53, but not wt p53. ${ }^{32,33}$

YAP was originally identified as a protein interacting with the c-Yes tyrosine kinase. ${ }^{34}$ Subsequent studies demonstrated that YAP is a transcription cofactor without direct DNA-binding activity. ${ }^{35}$ In addition to its interactions with p53 family members, YAP modulates transcription by binding to other transcription factors. ${ }^{36,37}$ Importantly, TEAD family transcription factors, which bind directly to specific DNA sequences and anchor YAP to chromatin, mediate most of the cell proliferative and anti-apoptotic transcriptional output of YAP and its paralog TAZ. ${ }^{38,39}$

YAP and TAZ are phosphorylated and inactivated by the serine/threonine kinases LATS1 and LATS2 within the Hippo pathway. ${ }^{40,41}$ In mammals, the core cascade of the canonical

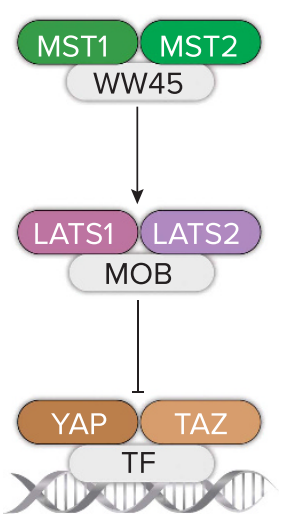

Figure 1 Simplified version of the key factors within the Hippo signaling pathway. The core of the Hippo pathway consists of MST1 and MST2, members of the Ste20 group of protein kinases; LATS1 and LATS2, members of the AGC kinase family, as well as their adaptor proteins Salvador (WW45) and MOB1, respectively. Activated LATS1/2 kinases phosphorylate the transcriptional co-factors YAP and TAZ on different sites, leading to their inactivation by cytoplasmic sequestering and proteasome-mediated degradation. TF, transcription factor

Hippo pathway involves the mammalian sterile 20-like kinases MST1/2 (MST), which can phosphorylate and activate the two large tumor suppressors, LATS1 and LATS2 (LATS) kinases. ${ }^{42,43}$ This kinase module is bolstered by two adaptor proteins, WW45 and MOB1 (shown schematically in Figure 1). A plethora of upstream signals modulate this kinase module, facilitating diverse biological outcomes. ${ }^{42}$ Although the core cascade is evolutionarily conserved, ${ }^{44,45}$ the wide array of biological processes modulated by each component suggests that they elicit their biological impact also through additional pathways. ${ }^{46-48}$ For instance, cytoskeleton dynamics and mechanical cues are prominent regulators of MST/LATSindependent $^{49}$ YAP/TAZ activity, which may liberate the kinases for interactions with the p53 pathway or other signaling pathways.

In this review, we examine the cross-talk between the Hippo and p53 pathways in an attempt to better understand mutual modulation and integration of function. The major interactions and biological outcomes are summarized in Table 1 and are detailed in the following sections. Although the specifics differ within different biological contexts, it is clear that the two pathways are strongly intertwined at multiple levels. Interestingly, in multiple cases, cross-talk between specific components may lead to different, sometimes contradicting, cellular outcomes. Similarly, deregulation of either pathway, as often occurs in cancer, has profound effects on the reciprocal pathway and, by extension, on cell fate.

\section{Stem Cells and Development}

A robust network of molecular regulation maintains embryonic stem cell (ESC) homeostasis. Signaling through Hippo and p53 pathways has important roles in dictating the fine balance of proliferation and differentiation of stem cells. For example, LATS kinases participate in maintaining the balance between pluripotency and differentiation, through both p53-dependent and -independent mechanisms. Inhibition of Lats1 and Lats2 expression in early mouse embryos results in irreversible 
Table 1 Points of cross-talk between the Hippo and p53 pathways

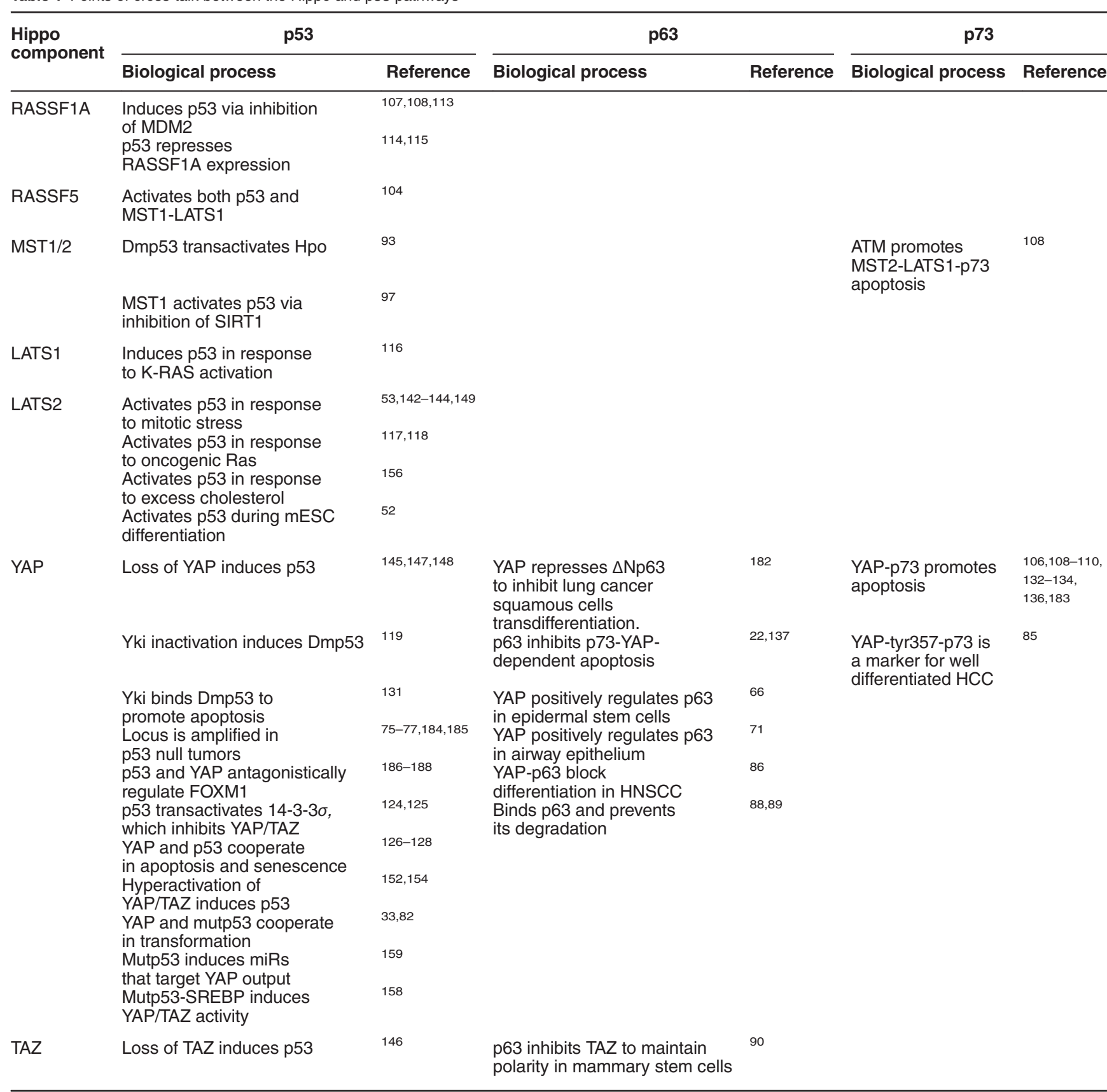

The table summarizes studies showing functional interactions between Hippo core components and different p53 family members

lineage misspecification and aberrant polarization of the inner cell mass, because of aberrant cellular localization of YAP. ${ }^{50}$ Furthermore, LATS2 is critical for blocking reprogramming of iPSCs by antagonizing the YAP paralog TAZ. ${ }^{51}$ Although the ability of LATS2 to restrict reprogramming is p53 independent, the functional interaction between LATS2 and p53 is necessary to maintain ESC homeostasis. ${ }^{52,53}$ Mouse ESCs (mESCs) lacking Lats2 are deficient in both sustaining pluripotency and responding to differentiation signals; 52 hand-in-hand with this is an inability to fully activate p53 during differentiation. Similarly, during normal development,
Lats2 and p53 engage in a positive feedback loop to ensure terminal differentiation. ${ }^{52}$

Subsequent to their impact on ESC differentiation, the p53 and Hippo pathways also have critical roles during development, when embryos are threatened by genotoxic stress. BRCA2 and Aurora A engage the Hippo pathway during replication fork stalling and mitosis, respectively, to ensure high fidelity cell division. ${ }^{54,55}$ Deletion of either gene in mice is embryonic lethal. Importantly, concurrent depletion of p53 accords partial rescue of lethality, ${ }^{56,57}$ suggesting an epistatic relationship of these genomic integrity genes during 
embryonic development. The core Hippo kinases and p53 act in parallel also in development to restrict organ size. ${ }^{58-60}$ For instance, conditional embryonic ablation of Yap or overexpression of p53 in mouse kidneys similarly causes underdevelopment and small organ size. ${ }^{61,62}$

In adult organisms, stem cell maintenance is required to sustain long-term preservation of tissue homeostasis. The mammalian epidermis is a rapidly regenerating epithelial tissue whose maintenance depends on the self-renewing ability of epidermal stem cells residing in the basal layer. ${ }^{63}$ Both p63 and YAP have essential roles in epidermal basal stem cell proliferation and epithelial stratification, and are considered epidermal stem cell markers. ${ }^{64-68}$ Accordingly, activation of YAP increases the number of cells expressing p63 and expands the epidermal stem cell compartment. ${ }^{66}$ One factor that may mediate YAP-p63 cross-talk is 14-3-30; downregulation of $14-3-3 \sigma$, which normally restrains YAP transcriptional activity by sequestration of phospho-Ser127 YAP in the cytoplasm, ${ }^{41}$ results in the expansion and immortalization of primary keratinocytes expressing p63. ${ }^{68,69}$ Interestingly, the $\Delta \mathrm{Np63}$ isoform can repress $14-3-3 \sigma$ expression. ${ }^{70}$ Together, these data suggest mechanisms by which YAP and p63 might augment each other's function.

In the lung, YAP and $\triangle \mathrm{Np} 63$ functionally interact to regulate airway epithelial stem cell homeostasis. ${ }^{71}$ YAP regulates differentiation of adult lung epithelium progenitors ${ }^{72}$ by binding $\triangle \mathrm{Np63}$ to drive the expression of common target genes, including $\triangle$ Np63 itself. $^{71,72}$ Persistent YAP expression is required to restrain adult airway basal stem cell differentiation and following injury YAP is upregulated in the regenerative tissue. ${ }^{73}$ Accordingly, loss of either YAP or p63 is associated with an excess of differentiated ciliated cells. p63 null mutants die at birth without basal progenitors in their airway epithelium. ${ }^{74}$ Similarly, conditional deletion of Yap from fetal lung epithelial progenitors reduces p63-positive cells and disrupts branching morphogenesis, resulting in lung hypoplasia. $^{72}$

Thus, proper regulation of YAP-p63 signaling maintains the steady-state stem cell pool through balanced rates of stem cell self-renewal and differentiation. Disparity of either of these processes can set the stage for tumorigenesis.

\section{Cancer Stem Cells}

Elevated expression of YAP may cooperate with loss of p53 to promote the emergence of cancers with an altered differentiation status, relative to their cell of origin. Thus, p53 deletion in mouse mammary gland luminal cells leads to clonal expansion without compromising luminal identity. ${ }^{75}$ However, subsequent mammary tumors often undergo luminal-to-basal transition in conjunction with amplification of the Yap gene. ${ }^{75}$ Additional studies also confirm that inactivation of p53, or combined loss of p53 and Rb, in mammary epithelium results in mammary carcinomas that bear recurrent Yap amplifications. ${ }^{76,77}$ These carcinomas become 'addicted' to YAP overexpression ${ }^{76}$ and harbor features of EMT and stem cell-like transcriptional signatures. ${ }^{77}$ In line with this notion, amplification of the Yap genomic region is among the top ranked amplification events in mouse models of prostate cancer driven by $\mathrm{p} 53 / \mathrm{PTEN}$ loss. ${ }^{78}$
Stem cell-like transcriptional signatures are often associated with the acquisition of stem-like properties, in that a subpopulation of transformed cells attains the ability to self-renew and generate the diverse types of cells that comprise the tumor. ${ }^{79}$ Cancer stem cells (CSCs), or tumor-initiating cells (TICs), have been identified in a wide variety of solid tumors and, analogous to embryonic and adult stem cells, both p53 family members and the Hippo components have been implicated as central regulators. TAZ is required for selfrenewal and tumor initiation capacities in breast CSCs. ${ }^{80}$ Similarly, mutation of p53 is sufficient to instigate a CSC-like phenotype. ${ }^{81}$ Recently, Escoll and colleagues ${ }^{82}$ have demonstrated that mutp53 enhances YAP/TAZ stability by regulating the WASP-interacting protein, WIP, to promote CSC properties in breast cancer and glioblastoma. This study provides one mechanism to explain the functional link between mutp53 and YAP/TAZ during tumorigenesis.

Similarly, YAP inhibition restores hepatocyte differentiation in advanced hepatocellular carcinoma (HCC).$^{83}$ Interestingly, different post-translational modifications, which have been shown to govern YAP stability, may dictate the differentiation status of HCC. ${ }^{84,85}$ Although hyperactivity of the YAP-TEAD functional axis predicts poor prognosis and stemness characteristics in HCC, tumors in which YAP is modified to interact with p73 ( $p$-Y357) display more apoptotic markers and have better prognosis. ${ }^{85}$ Thus, YAP can promote either tumor cell death or tumor-initiating properties, depending on its regulatory interactions with different members of the p53 family. In the next sections, we will review in more detail the YAP-p73 pro-apoptotic module and try to delineate the mechanisms that modulate the apparently opposing cellular functions of YAP.

p63 was also shown to engage with YAP to promote tumorigenesis. In head and neck squamous cell carcinoma (HNSCC), p63 collaborates with the chromatin-remodeling factor ACTL6A to block differentiation and drive regenerative proliferation in a YAP-dependent manner. ${ }^{86}$ Mechanistically, p63 represses the WWC1 (Kibra) promoter. As WWC1 encodes a cytosolic phosphoprotein that activates LATS kinases, ${ }^{87}$ p63-dependent silencing of WWC1 activates YAP. In line with this, upregulation of YAP transcriptional targets is correlated with poor prognosis of HNSCC. ${ }^{86}$ In addition to activation of YAP by p63, YAP binds $\triangle N p 63$, not only in developmental settings but also in a tumorigenic context. ${ }^{88,89}$ Here, YAP binding leads to $\Delta \mathrm{Np} 63$ stabilization by competition with the ITCH E3 ligase. ${ }^{88}$ Furthermore, the co-protective YAP- $\triangle$ Np63 association promotes epidermal squamous cell carcinoma CSC survival and migratory phenotypes. ${ }^{89}$ Interestingly, this interaction, like the YAP-mutp53 functional interaction, ${ }^{82}$ is regulated by membrane-associated integrin signaling. ${ }^{89}$

In contrast to the oncogenic $\Delta \mathrm{Np} 63$ isoform, which in some cases commandeers YAP to augment CSC properties, TAp63 and Hippo signaling seem to collaborate as tumor suppressors. In healthy tissue, TAp63 restricts mammary stem cell potential by inhibiting TAZ activity. ${ }^{90}$ Mechanistically, LKB1, a transcriptional target of $\mathrm{p} 63,{ }^{91}$ phosphorylates the microtubule affinity-regulating kinase family to safeguard LATS kinase activity. ${ }^{92}$ In aggressive human mammary adenocarcinomas, loss of TAp63 depolarizes cells and activates TAZ (but not 
YAP), which endows self-renewal capacity to breast cancer cells. ${ }^{80}$

In summary, oncogenic YAP/TAZ often exploit functional deficiencies in the p53 family of tumor suppressors to propel stem-like characteristics in cancers. Furthermore, tumors that harbor mutp53 or $\triangle \mathrm{Np63}$ rely on YAP/TAZ to reinforce the stem cell properties and elude death.

\section{Regulation of Apoptosis}

Cells bearing potentially transforming genomic aberrations must be eliminated to forestall tumorigenesis. The extent to which the Hippo tumor suppressors realize their anticancer function is often dependent on p53 or p73 activity.

Transcriptional control of MST-driven apoptosis is conserved from Drosophila to mammals, with p53 serving as a central mediator throughout evolution. In flies, Hpo (the MST ortholog) is activated by ionizing radiation in a Drosophila melanogaster p53 (Dmp53)-dependent manner (Figure 2). In fact, ectopic expression of Dmp53 is sufficient to activate Hpo and elicit cell death. ${ }^{93}$ Induction of apoptosis by both $\mathrm{Hpo}$ and Dmp53 relies on inhibition of the Drosophila inhibitor of apoptosis protein, DIAP1. In response to radiation, Dmp53 transactivates the Reaper locus, which encodes DIAP1 inhibitors such as Hid, grim and reaper itself. ${ }^{94}$ In addition, Hpo negatively regulates DIAP1 both by modulating the transactivation capacities of Yki (the fly YAP ortholog) and by directly phosphorylating and inducing the ubiquitin-dependent degradation of DIAP1. ${ }^{95,96}$

In mammals, the MST kinases facilitate apoptosis predominantly through transcription-independent mechanisms. MST1 augments apoptosis by phosphorylating and inhibiting the deacetylase SIRT1, thus enhancing p53 acetylation and function $^{97}$ (Figure 2). Interestingly, SIRT1 is regulated by multiple kinases, such as $\mathrm{CK} 2,{ }^{98}$ cyclin $\mathrm{B} / \mathrm{Cdk} 1^{99}$ and DYRK1A, ${ }^{100}$ kinases regulated collectively by LATS kinases, ${ }^{101-103}$ implicating a multi-pronged strategy by which Hippo kinases impact p53 activity.

In flies, as well as in mammals, p53 and MST share upstream regulatory factors. Overexpression of the Ras association domain family 5 (RASSF5) activates both the MST1-LATS1 and p53 axes. ${ }^{104}$ Another RASSF member, RASSF1A, promotes p53 family and MST activation in response to genotoxic stress. ${ }^{105-107}$ DNA damage-induced phosphorylation of RASFF1A by ATM promotes MST2-LATS1 activation and p73 stabilization. ${ }^{108}$ Mechanistically, RASSF1A promotes MST2 activation by abrogating the RAF1-MST2 inhibitory interaction. ${ }^{106}$ Subsequently, RASSF1A, together with the activated MST2-LATS1 complex, mediates apoptosis by inducing p73 transcription. ${ }^{108-110}$ Although the RASSF1AMST-WW45 (SAV1) ${ }^{111}$ complex can regulate p73 activity, it has also been suggested that RASSF1-WW45 can regulate p73 independently of the MST-LATS module. ${ }^{112}$ In addition to activating the MST and p73 pro-apoptotic arms, RASSF1A also stabilizes p53 by promoting MDM2 degradation. ${ }^{107}$ Indeed, polymorphisms of RASSF1, in the region encoding the recognition site for ATM phosphorylation, convey resistance to DNA damage-inducing agents and are associated with decreased p53 stability and poor patient prognosis. ${ }^{108,113}$ Surprisingly, wt p53 can bind and repress RASFF1A by facilitating its promoter hypermethylation ${ }^{114,115}$ (Figure 2). Whether this represents an inherent negative feedback loop between p53 and RASFF1A, or a pro-survival function that is conferred upon wt p53 in the course of tumor progression, remains to be determined.

Upstream modulation by RASSF proteins suggest a general sensitivity of the Hippo and p53 tumor-suppressor pathways to RAS-related signals. Indeed, aberrant hyperproliferation of cells caused by RAS oncogenic activation is a robust trigger of the p53-Hippo functional axis. Mutant K-RAS-dependent activation of the MST2-LATS1 kinase cassette results in sequestration of MDM2 by LATS1 and subsequent stabilization and activation of $\mathrm{p} 53^{116}$ (Figure 2). Similarly, H-RAS triggers LATS2 nuclear translocation and an increase in LATS2 protein levels, which leads to p53 activation. ${ }^{117}$ LATS2p53-dependent apoptosis upon oncogenic stress is mediated by phosphorylation of the apoptosis-stimulating protein of P53 1 (ASPP1), which shunts p53 to the promoters of proapoptotic genes. ${ }^{118}$ In transformed tissue, MST proapoptotic function is subverted by the ability of mutant

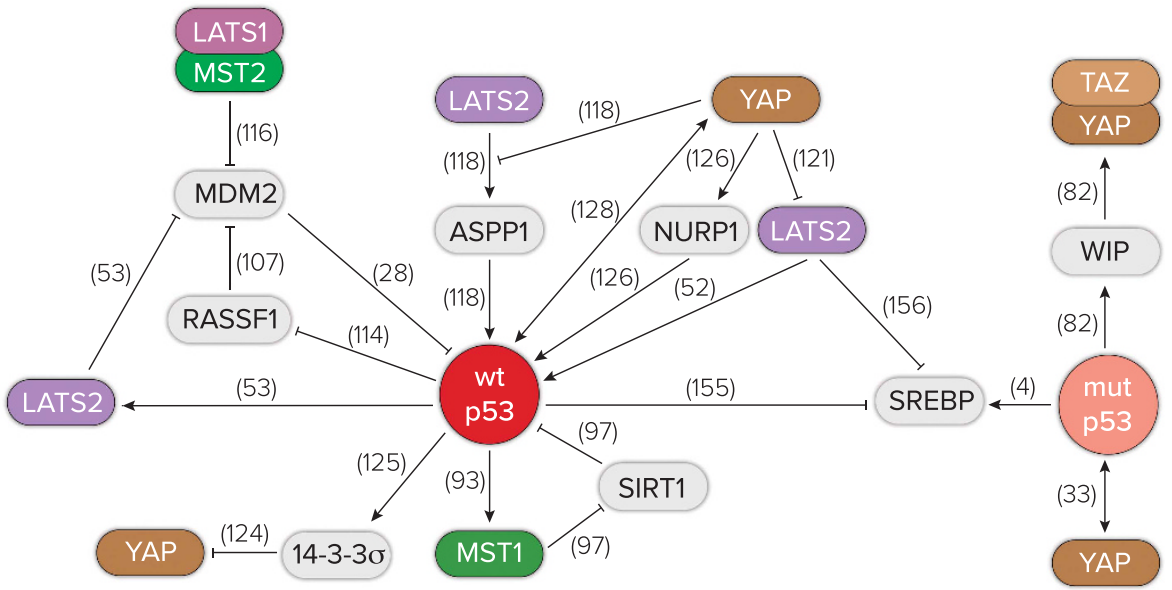

Figure 2 Scheme of reported cross-talk between core Hippo pathway components and wt (left) or mutant (right) p53. Both wt and mutp53 functionally interact with various components of the Hippo pathway (solid filled ovals) either directly or via additional factors (gray shaded ovals). Numbers above lines refer to publications describing the indicated regulatory vector 
K-RAS to divert wt K-RAS signaling. ${ }^{116}$ Similarly, H-RASdriven transformation entails a reduction of LATS2 levels in order to overcome p53-dependent apoptosis and senescence. ${ }^{117}$ Thus, during RAS-driven transformation there is a strong selective pressure to deregulate Hippo signaling in order to overcome p53-related tumor-suppressive functions.

Similar to oncogenic RAS, high YAP activity may disrupt the Hippo-p53 tumor-suppressive arm. In both flies and mammals, YAP expression overrides the ability of LATS and ASPP to drive p53-dependent transcription of pro-apoptotic genes. In Drosophila, Yki activation facilitates tissue growth by inhibiting p53 and ASPP, thereby repressing the expression of the proapoptotic gene reaper during tissue growth. ${ }^{119}$ In mammals, binding of cytoplasmic ASPP1 to LATS1 releases YAP/TAZ from negative regulation. ${ }^{120}$ Subsequently, the increased nuclear activity of YAP/TAZ inhibits apoptosis under low serum conditions and protects cells from anoikis. Overexpression of YAP can also inhibit apoptosis by disrupting the LATS2-ASPP1-p53 pro-apoptotic cascade ${ }^{118}$ (Figure 2). Similarly, nuclear YAP can repress LATS2 expression to inhibit replication stress-induced senescence mediated by $\mathrm{p53}$ dependent induction of p21. ${ }^{121}$ However, under different conditions, the YAP-TEAD complex can actually bind the LATS2 promoter to augment its transcription, ${ }^{122,123}$ suggesting that differential binding of YAP to various cofactors may differently modulate LATS2-p53 activity. Interestingly, this mode of regulation may be bidirectional, also enabling p53 to exert anti-YAP activities, as treatment with the YAP inhibitor Verteporfin causes sequestration of YAP in the cytoplasm, which is mediated by p53-dependent transcriptional induction of $14-3-3 \sigma .{ }^{124,125}$

In contrast to the above-described antagonistic roles of YAP and p53, there are apoptotic conditions in which YAP and p53 family members cooperate. In highly malignant tumorrepopulating cells, which are enriched by growing cancer cells on a soft matrix, the expression of nuclear protein 1 (NURP1) is compromised by reduced nuclear localization of YAP. ${ }^{126}$ Reduction in NURP1 expression is followed by a reduction in p53 mRNA and protein levels, suggesting an antitumorigenic axis involving YAP-NUPR1-p53. Similarly, YAP increases the sensitivity of hepatocellular and renal carcinoma cells to chemotherapy by increasing p53 expression and activity. $^{127,128}$ Interestingly, p53 can also drive a positive feedback, to transcriptionally increase YAP expression; indeed, YAP was recently identified as a direct transcriptional target of p53 in a genome-wide characterization of putative p53 response elements ${ }^{129}$ (Figure 2). Finally, YAP can facilitate p53-dependent cell death by promoting the stabilization of the pro-apoptotic factor ASPP2. ${ }^{130}$

In addition to modulation of p53 activity, YAP, as a transcriptional co-activator, can drive apoptosis by cooperating with p73. This functional interaction may be strongly conserved over evolution. In Drosophila, Yki interacts with the ancestral single p53 gene, Dmp53, to induce apoptosis. ${ }^{131}$ As Dmp53 resembles a p63/p73 hybrid gene, this may be analogous to mammalian YAP binding to $\mathrm{p} 73$, protecting p73 from ITCH-dependent degradation and driving apoptosis. ${ }^{132,133}$ YAP also enhances p73 transcriptional function by promoting p300-mediated p73 acetylation and recruitment to the promoters of apoptotic genes. ${ }^{106,110,134}$ Of note, RASSF1 promotes binding of YAP to $p 73$, in preference over other transcription factors such as TEAD and RUNX2. ${ }^{135}$ Thus, under specific regulatory cues, YAP has a central role in the cellular response to DNA damage by augmenting p73 stability and driving pro-apoptotic transcription. In tumors retaining wt $p 53$, curtailing of the tumor-suppressive YAP-p73 axis may be particularly crucial. Supporting this notion, reexpression of YAP in YAP-deficient multiple myeloma cells drives apoptosis by increasing p73 (not p53) levels and activity. ${ }^{136}$ Interestingly, YAP and p73 are inhibited, transcriptionally and post-translationally, by $\triangle \mathrm{Np} 63,{ }^{22,137}$ delineating an additional layer of cross-regulation between the pathways.

\section{Senescence and Ploidy}

The role of apoptosis as a tumor-suppressive mechanism is intuitive: it irreversibly removes dangerously transformed cells from the replicative pool. However, in other instances, senescence is the cell's method of choice to prevent proliferation of pre-malignant cells. ${ }^{138}$ One strong trigger for senescence is the genotoxic acquisition of aneuploidy, ${ }^{139}$ which can occur when chromosomes fail to properly segregate during mitosis. ${ }^{140}$ Numerical changes in whole chromosomes often precede transformation and are powerful inducers of p53. ${ }^{140,141}$ In response to mitotic stress, LATS2 binds and inhibits MDM2, to enable p53 activation and induce G1/S arrest. In turn, activated p53 transcriptionally induces LATS2 expression, creating a positive feedback loop to ensure that the cell maintains proper DNA content ${ }^{53,142}$ (Figure 2). The ability of LATS2 to sense extra chromosomes has been attributed to changes in the actin cytoskeleton, specifically to the reduction of RHOA activity. ${ }^{143}$ Indeed megakaryocytes, naturally occurring polyploid cells, in which polyploidization is needed for platelet formation, escape the p53-LATS axis by uncoupling RHOA activity from Hippo signaling. ${ }^{144}$ Thus, induction of LATS2 in response to ploidy aberrations enables simultaneous activation of a protective p53-dependent growth arrest and inactivation of YAP/TAZ pro-proliferative signals.

LATS2 and p53 cooperate also in response to replication stress, to induce p21 expression and promote cellular senescence. ${ }^{117,121}$ This p53-LATS homeostatic axis is targeted by oncogenes to overcome tumor-suppressive checkpoints and increase tolerance to polyploidy. For instance, silencing of YAP/TAZ in cancer or stem cell lines facilitates p53 activation and growth arrest. ${ }^{145-148}$ Similarly, v-SRC, a viral mutant variant of the c-SRC cellular proto-oncogene, enables abnormal spindle formation and transformation by reducing p53 levels and promoting YAP nuclear localization by squelching LATS activity. ${ }^{149}$ An analogous situation occurs during $\mathrm{H}-\mathrm{RAS}$ transformation, in which silencing of LATS2 permits proliferation of polyploid cells. ${ }^{117}$

On the other hand, perhaps akin to the above-mentioned p53-dependent oncogene-induced senescence, ${ }^{150,151}$ hyperactivation of YAP/TAZ can also activate p53. Loss of WRN protein function, as seen in the premature aging Werner syndrome, results in cellular senescence. ${ }^{152}$ In cancer models, depletion of WRN decreases tumor growth and increases chemotherapy sensitivity. ${ }^{153}$ Mechanistically, the depletion of WRN induces ATM-dependent phosphorylation of YAP and p53, leading to YAP stabilization and p53-dependent 
senescence. ${ }^{152}$ Similarly, in the liver, YAP/TAZ hyperactivation leads to DNA damage and the generation of polyploid cells, which induces p53 and leads to hepatocyte senescence. ${ }^{154}$

\section{Metabolic Homeostasis}

The sterol regulatory element-binding protein (SREBP) transcription factors are master regulators of cholesterol and lipid homeostasis and are emerging as central factors in the cross-talk between p53 and the Hippo pathway. SREBP1 expression is transcriptionally repressed by wt p53. ${ }^{155}$ LATS2 acts in concert with p53 to inhibit SREBP function (Figure 2) by binding the ER-tethered precursors of SREBP1 and SREBP2, impeding their processing and quenching the subsequent transcriptional activity of the cleaved, nuclear SREBPs. ${ }^{156}$ Expression of hepatic Lats2 is required to avoid fatty liver disease and for induction of a p53 pro-apoptotic response when mice are challenged with excess dietary cholesterol. ${ }^{156}$ Conversely, forced expression of SREBP1 in mouse liver leads to p53 activation. ${ }^{157}$ Thus, together, p53 and LATS2 function to maintain appropriate levels of SREBP activity and cholesterol homeostasis within the liver.

Activation of the mevalonate-cholesterol pathway promotes YAP/TAZ nuclear localization and transcriptional activity by activation of $\mathrm{RHO}$ GTPases. ${ }^{158}$ Contrary to wt p53 repression of SREBP, mutp53 cooperates with SREBP to promote the expression of sterol-related genes ${ }^{4}$ and increases YAP/TAZdependent gene expression ${ }^{158}$ (Figure 2). Accordingly, expression data from breast cancer patients show that both SREBP and YAP/TAZ-related genes are expressed at higher levels in mutp53 tumors compared with wt p53 tumors. ${ }^{158}$ Of note, in this case the regulation of YAP/TAZ by SREBP activation is LATS independent.

In line with the epistatic regulation of YAP and mutp53, recently $\mathrm{Di}$ Agostino and colleagues $^{33}$ demonstrated a physical interaction between YAP (but not TAZ) and mutp53 in breast cancer cell lines (Figure 2). This interaction drives the expression of pro-proliferative genes such as cyclin $A$ (CCNA), cyclin B (CCNB) and CDK1 to promote tumorigenesis. Mutp53 may also augment YAP/TAZ activity by deregulating upstream Hippo components. Indeed, the miRNA transcriptome associated with mutp53 substantially differs from that of wt p53, and is significantly enriched with miRNAs that putatively target Hippo pathway components. ${ }^{159}$

Tumors must coordinate the rewiring of cell hyperproliferation with the altered metabolic demands of oncogenesis. To unleash cells' pro-proliferative potential, mutp53 and YAP converge on additional facets of cell metabolism, beyond the aforementioned mevalonate pathway. For instance, tumorassociated mutp53 stimulates the Warburg effect through promoting GLUT1 translocation to the plasma membrane, which is mediated by activated RHOA and its downstream effector ROCK. ${ }^{5}$ Not only is RHOA a well-documented upstream effector of the Hippo pathway, ${ }^{49}$ but also augmented glycolysis has been shown to be crucial to sustain YAP/TAZ transcriptional activity and pro-tumorigenic functions. ${ }^{160-163}$ Similarly, both YAP and mutp53 protein levels are dependent on nutrient availability. Intriguingly, glucose restriction selectively promotes both mutp53 and YAP degradation through autophagy. ${ }^{164,165}$ Moreover, the recent discovery that the MST kinases sustain autophagosome formation by phosphorylating $L C 3^{166}$ suggests that cross-talk between Hippo signaling, autophagy and mutp53 might function at multiple junctions.

Ultimately, homeostasis represents a balance between stemness and differentiation, cell death and proliferation, and anabolism and catabolism. Given the important functions of the Hippo and p53 pathways in these processes, it is no wonder that they are profoundly intertwined.

\section{Conclusions}

The complexity of signaling pathways tends to increase over evolution, facilitated at least partially by gene duplications events, as seen for both the p53 family members and Hippo pathway components. ${ }^{14,44}$ Paralogs may provide a platform to increase and fine tune the integration of signaling pathways into a comprehensive and precise network that maintains homeostasis and fitness. In the case of the p53 and Hippo pathways, signaling integration is highly conserved, with multiple interaction nodes in organisms with no duplications of either TP53 or Hippo genes (i.e., Drosophila). Interestingly, a significant portion of the cross-talk between the two pathways is dedicated to maintaining homeostasis between stemness and differentiation. This may be related to the primordial and conserved role of Hippo components and p53 family members in germline protection and organismal development. ${ }^{14,167}$ Alterations in this balance may be hijacked by cancer cells to maintain stemness features and to increase their competitive advantage.

Coordination of the p53 and Hippo tumor-suppressive pathways occurs on multiple levels (simplified summary in Table 1). For example, LATS2 can bind directly to MDM2, the major negative regulator of p53, blocking the ability of MDM2 to target p53 for ubiquitin-dependent proteasomal degradation and resulting in p53 accumulation and activation. ${ }^{53}$ Through this capacity, LATS2 mediates p53 activation in response to a number of diverse types of stress signals, including mitotic apparatus dysfunction, expression of oncogenic RAS and cholesterol overload, ${ }^{53,117,118,156}$ and during ESC differentiation. ${ }^{52}$ Similarly, both YAP and TAZ collaborate with different p63 isoforms to modulate adult and CSCs, ${ }^{71,86,89,90}$ whereas MST and YAP cooperate to direct p73-dependent apoptosis. ${ }^{106,168}$ mutp53 physically interacts with YAP to coordinately regulate the expression of cancer-related genes. ${ }^{33}$ In turn, optimal activity of YAP and TAZ relies on products of the mevalonate pathway, ${ }^{158}$ which is regulated positively by mutp53 ${ }^{4}$ and negatively by LATS2. ${ }^{156}$ Importantly, downregulation of LATS alters the properties of wt p53. This is reflected by reduced phosphorylation, conformational changes and alteration of transcriptional targets, all of which contribute to a mutant-like phenotype of wt p53. ${ }^{169}$

These multiple points of regulatory interactions may also dictate selective forces during tumorigenesis that drive rewiring and alterations of the two pathways. Although the TP53 gene is highly mutated in diverse cancer types, pancancer TCGA data ${ }^{170}$ reveal that there are also tumor types with relatively low prevalence of such mutations, for example, kidney renal clear-cell carcinoma 3\%; lymphoblastic acute myeloid leukemia (LAML) 8\%; glioblastoma multiforme $29 \%$, as opposed to cancers with frequent TP53 mutations (ovarian 


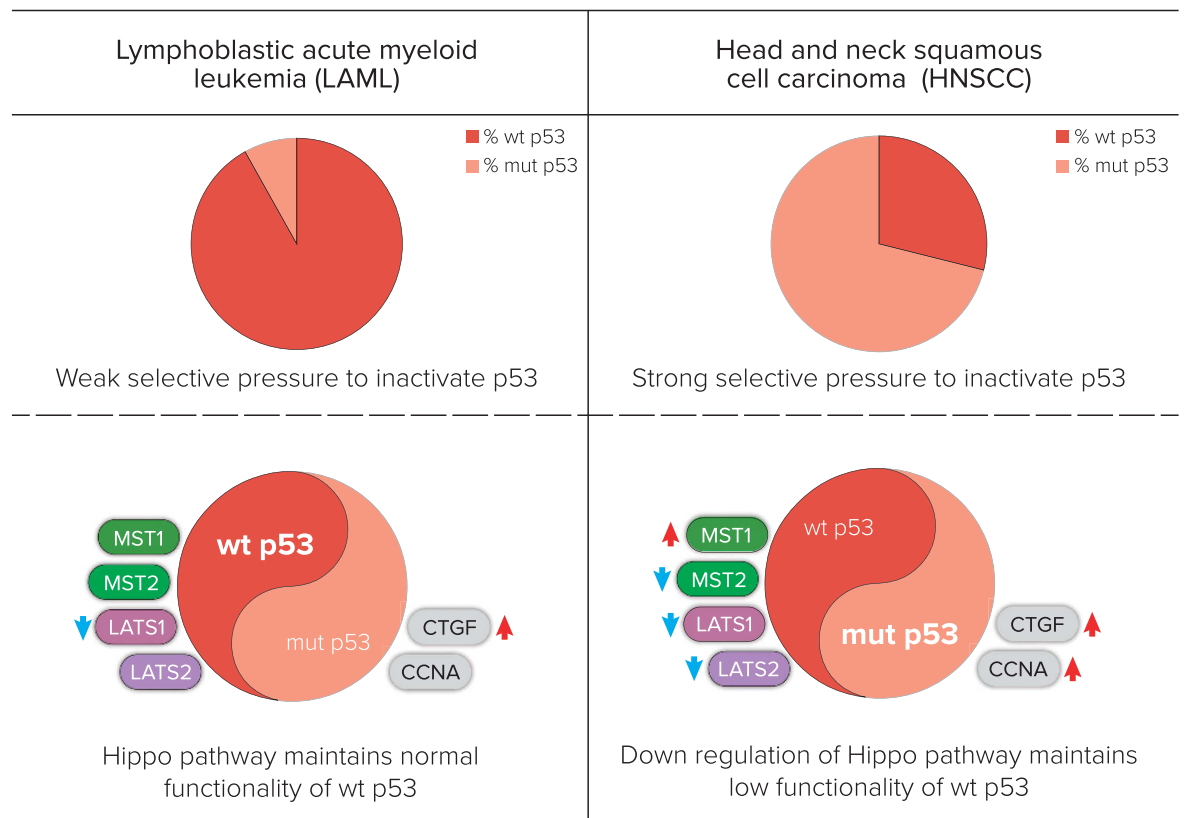

Figure 3 Different types of cancers have distinctive patterns of Hippo-p53 axis inactivation. The pressure to disable wt p53 (wt p53) function in human tumors is associated with deregulation of Hippo pathway components. LAML (left panel) represents a cancer type with a low percentage of TP53 mutations, whereas HNSCC (right panel) represents a tumor type harboring a high percentage of TP53 mutations. For both cancer types, the portion of wt and mutp53 cases are shown in the upper section (TCGA data). Within each cancer type, expression of core Hippo components (TCGA data) was compared between tumors with wt or mutp53. Statistically significant (FDR $<0.1)$ upregulation (red arrow) or downregulation (blue arrow) is indicated on the left or right of the yin-yang symbol, for cases with wt and mutp53, respectively

carcinoma $83 \%$, lung squamous cell carcinoma $82 \%$, HNSCC $71 \%$ ). Comparing the expression levels of the core Hippo components in tumors with wt or mutp53 within each type of cancer reveals an interesting trend (Figure 3). In tumor types where the pressure to mutationally inactivate p53 is low (exemplified by LAML), the large proportion of tumors that retain wt p53 also preserve expression of the Hippo tumorsuppressive kinase module. Furthermore, in the relatively few tumors with p53 mutations, the YAP-mutp53 target CCNA is not upregulated. Interestingly in HNSCC in which there is a strong pressure to mutate TP53, tumors that retain wt p53 appear to compromise its function by downregulating the Hippo kinases. Furthermore, in this tumor type, CCNA is expressed at higher levels in mutp53 tumors, suggesting the existence of a transcriptionally active mutp53-YAP complex. Although only correlative, these observations may reflect the close-knit network of interactions between the two pathways, and its corruption in the course of different malignant contexts.

There is growing evidence supporting a scenario in which incoming physiological signals dynamically dictate a variety of functions and conformational states for different proteins. These conformational states might generate a functional spectrum ranging from wt to mutant-like behavior ('pseudomutant'), even in proteins that are wt by sequence. For instance, specific post-translational modifications trigger changes in the conformation of wt p53 that can mediate its ability to interact with binding partners and fortify its commitment to specific biological processes. ${ }^{171}$ External and internal signaling events, such as growth factors, oxygen availability and differential binding to distinct DNA sequences or molecular chaperones also have an impact on the conformation of $\mathrm{p} 53$. $^{172-176}$

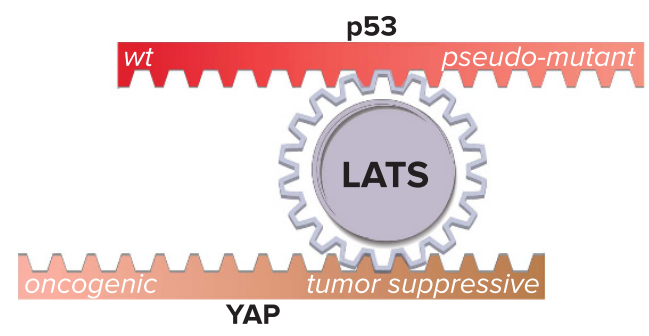

Figure 4 A LATS 'rheostat' may modulate p53 and YAP functionality. LATS1 and LATS2 (LATS) kinases are central modulators of both p53 and YAP activity. Although both p53 and YAP can facilitate both pro- and antitumorigenic activities, LATS kinases emerge as major regulators that maintain wt $p 53$ activity and balance the tumor promoting functions of YAP

Similarly, differential binding of YAP to TEAD or p73 reflects the diametrically opposing roles of YAP. Whereas YAP association with TEAD leads to cell proliferation, epithelialto-mesenchymal transition, inhibition of apoptosis and tumorigenesis, ${ }^{177}$ YAP binding to $\mathrm{p} 73$ promotes apoptosis and differentiation. ${ }^{106,110}$ Thus in different conditions, YAP can feature either as tumor suppressor or as oncogene. ${ }^{36}$

Both YAP and p53 might embody an inherent capacity to adopt pseudomutant status, which must be coordinated; perhaps by their common upstream regulator, LATS (Figure 4). Interaction of additional elements of the Hippo pathway beyond LATS, ${ }^{169}$ might also modulate $\mathrm{p} 53$ conformation. In turn, pseudomutant wt p53 might mimic the impact of mutp53 on Hippo pathway function. In parallel, YAP itself might embody a spectrum of conformations that facilitate tumorsuppressive or oncogenic biological outcomes, depending on various signaling alterations including p53's functional state as suggested recently by Ferraiuolo and colleagues. ${ }^{178}$ 
Conceivably, YAP, which has been shown to bind mutp53, might bind wt p53 in conditions that induce pseudomutant conformation.

This dynamic situation is at odds with our binary categorization of genes into 'oncogenes' or 'tumor suppressors'. When proteins are thus labeled, our prediction of the range of their potential is restricted. For instance, we typically assume that tumor suppressors will inherently favor cell death. However, seemingly paradoxical observations made in tumor patients, as well as mouse models indicate that excessive apoptosis actually can aggravate tumorigenesis. ${ }^{179,180}$ In contrast, through their ability to enable metabolic adaptation, p53 and Hippo components can support cell survival. These cell-protective functions help avoid damage and thereby prevent cancer development, ${ }^{181}$ but these same functions may eventually by hijacked by cancer cells to assure their wellbeing and provide them with a competitive advantage in stressful conditions. Understanding the nuances that control context-dependent protein behavior and contrasting physiological fates is not merely a basic science puzzle, but will be essential for efficient therapeutic implementations.

\section{Conflict of Interest}

The authors declare no conflict of interest.

Acknowledgements. Research in the authors' lab is made possible by the Dr. Miriam and Sheldon G Adelson Medical Research Foundation, a Center of Excellence grant (2084/15) from the Israel Science Foundation, a grant from the Robert Bosch Stiftung and funding received from the MINERVA Stiftung with the funds from the BMBF of the Federal Republic of Germany. Dr. Ron Rotkopf assisted with bioinformatic analyses of TCGA data. Ishai Sher helped with graphical illustrations.

1. Joerger AC, Fersht AR. The p53 pathway: origins, inactivation in cancer, and emerging therapeutic approaches. Annu Rev Biochem 2016; 85: 375-404.

2. Bieging KT, Mello SS, Attardi LD. Unravelling mechanisms of p53-mediated tumour suppression. Nat Rev Cancer 2014; 14: 359-370.

3. Muller PAJ, Vousden KH. p53 mutations in cancer. Nat Cell Biol 2013; 15: 2-8.

4. Freed-Pastor WA, Mizuno H, Zhao X, Langerød A, Moon S-H, Rodriguez-Barrueco R et al. Mutant p53 disrupts mammary tissue architecture via the mevalonate pathway. Cell 2012; 148: 244-258.

5. Zhang $C$, Liu J, Liang Y, Wu R, Zhao Y, Hong $X$ et al. Tumour-associated mutant p53 drives the Warburg effect. Nat Commun 2013; 4: 2935

6. Subramanian M, Francis P, Bilke S, Li XL, Hara T, Lu X et al. A mutant p53/let-7i-axisregulated gene network drives cell migration, invasion and metastasis. Oncogene 2015; 34 1094-1104.

7. Weissmueller S, Manchado E, Saborowski M, Morris JP, Wagenblast E, Davis CA et al. Mutant p53 drives pancreatic cancer metastasis through cell-autonomous PDGF receptor $\beta$ signaling. Cell 2014; 157: 382-394.

8. Do PM, Varanasi L, Fan S, Li C, Kubacka I, Newman V et al. Mutant p53 cooperates with ETS2 to promote etoposide resistance. Genes Dev 2012; 26: 830-845.

9. Vousden KH, Prives C. Blinded by the light: the growing complexity of p53. Cell 2009; 137 : 413-431.

10. Aylon Y, Oren M. The paradox of p53: what, how, and why? Cold Spring Harb Perspect Med 2016; 6 (doi:10.1101/cshperspect.a026328).

11. Scherz-Shouval R, Weidberg H, Gonen C, Wilder S, Elazar Z, Oren M. p53-dependent regulation of autophagy protein LC3 supports cancer cell survival under prolonged starvation. Proc Natl Acad Sci USA 2010; 107: 18511-18516.

12. Jänicke RU, Sohn D, Schulze-Osthoff K. The dark side of a tumor suppressor: antiapoptotic p53. Cell Death Differ 2008; 15: 959-976.

13. Bensaad K, Tsuruta A, Selak MA, Vidal MNC, Nakano K, Bartrons R et al. TIGAR, a p53inducible regulator of glycolysis and apoptosis. Cell 2006; 126: 107-120.

14. Belyi VA, Ak P, Markert E, Wang H, Hu W, Puzio-Kuter $A$ et al. The origins and evolution of the p53 family of genes. Cold Spring Harb Perspect Biol 2010; 2: a001198.

15. Mills AA, Zheng B, Wang $X J$, Vogel $H$, Roop DR, Bradley A. p63 is a p53 homologue required for limb and epidermal morphogenesis. Nature 1999; 398: 708-713.
16. Yang A, Schweitzer R, Sun D, Kaghad M, Walker N, Bronson RT et al. p63 is essential for regenerative proliferation in limb, craniofacial and epithelial development. Nature 1999; 398 714-718.

17. Yang A, Walker N, Bronson R, Kaghad M, Oosterwegel M, Bonnin J et al. p73-deficient mice have neurological, pheromonal and inflammatory defects but lack spontaneous tumours. Nature 2000; 404: 99-103.

18. Irwin MS, Kondo K, Marin MC, Cheng LS, Hahn WC, Kaelin WG. Chemosensitivity linked to p73 function. Cancer Cell 2003; 3: 403-410.

19. Suh E-K, Yang A, Kettenbach A, Bamberger C, Michaelis AH, Zhu Z et al. p63 protects the female germ line during meiotic arrest. Nature 2006; 444: 624-628.

20. Murray-Zmijewski F, Lane DP, Bourdon J-C. p53/p63/p73 isoforms: an orchestra of isoforms to harmonise cell differentiation and response to stress. Cell Death Differ 2006; 13 962-972.

21. Barbieri CE, Tang LJ, Brown KA, Pietenpol JA. Loss of p63 leads to increased cell migration and up-regulation of genes involved in invasion and metastasis. Cancer Res 2006; 66: 7589-7597.

22. Rocco JW, Leong C-O, Kuperwasser N, DeYoung MP, Ellisen LW. p63 mediates survival in squamous cell carcinoma by suppression of p73-dependent apoptosis. Cancer Cell 2006 9: 45-56.

23. Rosenbluth JM, Pietenpol JA. The jury is in: $\mathrm{p73}$ is a tumor suppressor after all. Genes Dev 2008; 22: 2591-2595.

24. Su X, Chakravarti D, Flores ER. p63 steps into the limelight: crucial roles in the suppression of tumorigenesis and metastasis. Nat Rev Cancer 2013; 13: 136-143.

25. Agami R, Blandino G, Oren M, Shaul Y. Interaction of c-Abl and p73alpha and their collaboration to induce apoptosis. Nature 1999; 399: 809-813.

26. Gong JG, Costanzo A, Yang HQ, Melino G, Kaelin WG, Levrero M et al. The tyrosine kinase c-Abl regulates p73 in apoptotic response to cisplatin-induced DNA damage. Nature 1999; 399: 806-809.

27. Bode AM, Dong Z. Post-translational modification of p53 in tumorigenesis. Nat Rev Cancer 2004; 4: 793-805.

28. Haupt $Y$, Maya R, Kazaz A, Oren M. Mdm2 promotes the rapid degradation of p53. Nature 1997; 387: 296-299.

29. Bálint $\mathrm{E}$, Bates $\mathrm{S}$, Vousden $\mathrm{KH}$. Mdm2 binds $\mathrm{p73}$ alpha without targeting degradation Oncogene 1999; 18: 3923-3929.

30. Rossi M, De Laurenzi V, Munarriz E, Green DR, Liu Y-C, Vousden KH et al. The ubiquitinprotein ligase Itch regulates p73 stability. EMBO J 2005; 24: 836-848.

31. Rossi M, Aqeilan RI, Neale M, Candi E, Salomoni P, Knight RA et al. The E3 ubiquitin ligase Itch controls the protein stability of p63. Proc Natl Acad Sci USA 2006; 103: 12753-12758.

32. Strano S, Munarriz E, Rossi M, Castagnoli L, Shaul Y, Sacchi A et al. Physical interaction with Yes-associated protein enhances p73 transcriptional activity. J Biol Chem 2001; 276: 15164-15173.

33. Di Agostino S, Sorrentino G, Ingallina E, Valenti F, Ferraiuolo M, Bicciato $S$ et al. YAP enhances the pro-proliferative transcriptional activity of mutant p53 proteins. EMBO Rep 2016; 17: 188-201.

34. Sudol M. Yes-associated protein (YAP65) is a proline-rich phosphoprotein that binds to the SH3 domain of the Yes proto-oncogene product. Oncogene 1994; 9: 2145-2152.

35. Yagi R, Chen LF, Shigesada K, Murakami Y, Ito YA. WW domain-containing yes-associated protein (YAP) is a novel transcriptional co-activator. EMBO J 1999; 18: 2551-2562.

36. Bertini E, Oka T, Sudol M, Strano S, Blandino G. YAP: at the crossroad between transformation and tumor suppression. Cell Cycle 2009; 8: 49-57.

37. Strano S, Fausti F, Di Agostino S, Sudol M, Blandino G. PML surfs into HIPPO tumor suppressor pathway. Front Oncol 2013; 3: 36.

38. Zhao B, Ye X, Yu J, Li L, Li W, Li S et al. TEAD mediates YAP-dependent gene induction and growth control. Genes Dev 2008; 22: 1962-1971.

39. Zhang H, Liu C-Y, Zha Z-Y, Zhao B, Yao J, Zhao S et al. TEAD transcription factors mediate the function of TAZ in cell growth and epithelial-mesenchymal transition. J Biol Chem 2009; 284: 13355-13362.

40. Lei Q-Y, Zhang H, Zhao B, Zha Z-Y, Bai F, Pei X-H et al. TAZ promotes cell proliferation and epithelial-mesenchymal transition and is inhibited by the hippo pathway. Mol Cell Biol 2008; 28: 2426-2436.

41. Zhao B, Wei X, Li W, Udan RS, Yang Q, Kim J et al. Inactivation of YAP oncoprotein by the Hippo pathway is involved in cell contact inhibition and tissue growth control. Genes Dev 2007; 21: 2747-2761.

42. Meng Z, Moroishi T, Guan K-L. Mechanisms of Hippo pathway regulation. Genes Dev 2016; 30: 1-17

43. Oren M, Aylon Y The Hippo Signaling Pathway and Cancer. illustrated. Springer Science \& Business Media 2013. (doi:10.1007/978-1-4614-6220-0)

44. Hilman D, Gat U. The evolutionary history of YAP and the hippo/YAP pathway. Mol Biol Evol 2011; 28: 2403-2417.

45. Zhao B, Li L, Lei Q, Guan K-L. The Hippo-YAP pathway in organ size control and tumorigenesis: an updated version. Genes Dev 2010; 24: 862-874.

46. Mauviel A, Nallet-Staub F, Varelas X. Integrating developmental signals: a Hippo in the (path)way. Oncogene 2012; 31: 1743-1756.

47. Thompson BJ, Sahai E. MST kinases in development and disease. J Cell Biol 2015; 210 871-882.

48. Furth N, Aylon Y. The LATS1 and LATS2 tumor suppressors: beyond the Hippo pathway. Cell Death Differ 2017; 24: 1488-1501. 
49. Dupont S, Morsut L, Aragona M, Enzo E, Giulitti S, Cordenonsi M et al. Role of YAP/TAZ in mechanotransduction. Nature 2011; 474: 179-183.

50. Lorthongpanich C, Messerschmidt DM, Chan SW, Hong W, Knowles BB, Solter D. Temporal reduction of LATS kinases in the early preimplantation embryo prevents ICM lineage differentiation. Genes Dev 2013; 27: 1441-1446.

51. Qin H, Blaschke K, Wei G, Ohi Y, Blouin L, Qi Z et al. Transcriptional analysis of pluripotency reveals the Hippo pathway as a barrier to reprogramming. Hum $\mathrm{Mol}$ Genet 2012; 21: 2054-2067.

52. Aylon Y, Sarver A, Tovy A, Ainbinder E, Oren M. Lats2 is critical for the pluripotency and proper differentiation of stem cells. Cell Death Differ 2014; 21: 624-633.

53. Aylon Y, Michael D, Shmueli A, Yabuta N, Nojima H, Oren M. A positive feedback loop between the $\mathrm{p} 53$ and Lats2 tumor suppressors prevents tetraploidization. Genes Dev 2006 20: 2687-2700.

54. Pefani D-E, Latusek R, Pires I, Grawenda AM, Yee KS, Hamilton G et al. RASSF1A-LATS1 signalling stabilizes replication forks by restricting CDK2-mediated phosphorylation of BRCA2. Nat Cell Biol 2014; 16: 962-9711.

55. Yabuta N, Mukai S, Okada N, Aylon Y, Nojima $H$. The tumor suppressor Lats2 is pivotal in Aurora A and Aurora B signaling during mitosis. Cell Cycle 2011; 10 : 2724-2736.

56. Ludwig T, Chapman DL, Papaioannou VE, Efstratiadis A. Targeted mutations of breas cancer susceptibility gene homologs in mice: lethal phenotypes of Brca1, Brca2, Brca1/Brca2, Brca1/p53, and Brca2/p53 nullizygous embryos. Genes Dev 1997; 11 1226-1241.

57. Lee D-F, Su J, Ang Y-S, Carvajal-Vergara X, Mulero-Navarro S, Pereira CF et al. Regulation of embryonic and induced pluripotency by aurora kinase-p53 signaling. Cell Stem Cell 2012; 11: 179-194.

58. Pan D. Hippo signaling in organ size control. Genes Dev 2007; 21: 886-897.

59. Mesquita D, Dekanty A, Milán M. A dp53-dependent mechanism involved in coordinating tissue growth in Drosophila. PLoS Biol 2010; 8: e1000566.

60. Heallen T, Zhang M, Wang J, Bonilla-Claudio M, Klysik E, Johnson RL et al. Hippo pathway inhibits Wnt signaling to restrain cardiomyocyte proliferation and heart size. Science 2011 332: 458-461.

61. Godley LA, Kopp JB, Eckhaus M, Paglino JJ, Owens J, Varmus HE. Wild-type p53 transgenic mice exhibit altered differentiation of the ureteric bud and possess small kidneys. Genes Dev 1996; 10: 836-850.

62. Reginensi A, Scott RP, Gregorieff A, Bagherie-Lachidan M, Chung C, Lim D-S et al. Yapand Cdc42-dependent nephrogenesis and morphogenesis during mouse kidney development. PLOS Genet 2013; 9: e1003380.

63. Fuchs E. Scratching the surface of skin development. Nature 2007; 445: 834-842.

64. Koster MI, Kim S, Mills AA, DeMayo FJ, Roop DR. p63 is the molecular switch for initiation of an epithelial stratification program. Genes Dev 2004; 18: 126-131.

65. Romano R-A, Ortt K, Birkaya B, Smalley K, Sinha S. An active role of the DeltaN isoform of p63 in regulating basal keratin genes $\mathrm{K} 5$ and $\mathrm{K} 14$ and directing epidermal cell fate. PLoS ONE 2009; 4: e5623.

66. Schlegelmilch K, Mohseni M, Kirak O, Pruszak J, Rodriguez JR, Zhou D et al. Yap1 acts downstream of $\alpha$-catenin to control epidermal proliferation. Cell 2011; 144: 782-795.

67. Zhang H, Pasolli HA, Fuchs E. Yes-associated protein (YAP) transcriptional coactivator functions in balancing growth and differentiation in skin. Proc Natl Acad Sci USA 2011; 108 2270-2275.

68. Pellegrini G, Dellambra E, Golisano O, Martinelli E, Fantozzi I, Bondanza S et al. p63 identifies keratinocyte stem cells. Proc Natl Acad Sci USA 2001; 98: 3156-3161.

69. Dellambra E, Golisano O, Bondanza S, Siviero E, Lacal P, Molinari M et al. Downregulation of 14-3-3sigma prevents clonal evolution and leads to immortalization of primary human keratinocytes. J Cell Biol 2000; 149: 1117-1130.

70. Westfall MD, Mays DJ, Sniezek JC, Pietenpol JA. The Delta Np63 alpha phosphoprotein binds the p21 and 14-3-3 sigma promoters in vivo and has transcriptional repressor activity that is reduced by Hay-Wells syndrome-derived mutations. Mol Cell Biol 2003; 23 . 2264-2276.

71. Zhao R, Fallon TR, Saladi SV, Pardo-Saganta A, Villoria J, Mou H et al. Yap tunes airway epithelial size and architecture by regulating the identity, maintenance, and self-renewal of stem cells. Dev Cell 2014; 30: 151-165.

72. Mahoney JE, Mori M, Szymaniak AD, Varelas X, Cardoso WV. The hippo pathway effector Yap controls patterning and differentiation of airway epithelial progenitors. Dev Cell 2014; 30: $137-150$

73. Lange AW, Sridharan A, Xu Y, Stripp BR, Perl A-K, Whitsett JA. Hippo/Yap signaling controls epithelial progenitor cell proliferation and differentiation in the embryonic and adult lung. J Mol Cell Biol 2015; 7: 35-47.

74. Daniely Y, Liao G, Dixon D, Linnoila RI, Lori A, Randell SH et al. Critical role of p63 in the development of a normal esophageal and tracheobronchial epithelium. Am J Physiol, Cell Physiol 2004; 287: C171-C181.

75. Tao L, Xiang D, Xie Y, Bronson RT, Li Z. Induced p53 loss in mouse luminal cells causes clonal expansion and development of mammary tumours. Nat Commun 2017; 8: 14431.

76. Cheng L, Zhou Z, Flesken-Nikitin A, Toshkov IA, Wang W, Camps J et al. Rb inactivation accelerates neoplastic growth and substitutes for recurrent amplification of cIAP1, cIAP2 and Yap1 in sporadic mammary carcinoma associated with p53 deficiency. Oncogene 2010; 29: 5700-5711.
77. Jones RA, Robinson TJ, Liu JC, Shrestha M, Voisin V, Ju Y et al. RB1 deficiency in triplenegative breast cancer induces mitochondrial protein translation. J Clin Invest 2016; 126: 3739-3757.

78. Wanjala J, Taylor BS, Chapinski C, Hieronymus H, Wongvipat J, Chen Y et al. Identifying actionable targets through integrative analyses of GEM model and human prostate cancer genomic profiling. Mol Cancer Ther 2015; 14: 278-288.

79. Visvader JE, Lindeman GJ. Cancer stem cells in solid tumours: accumulating evidence and unresolved questions. Nat Rev Cancer 2008; 8: 755-768.

80. Cordenonsi M, Zanconato F, Azzolin L, Forcato M, Rosato A, Frasson C et al. The Hippo transducer TAZ confers cancer stem cell-related traits on breast cancer cells. Cell 2011; 147: $759-772$.

81. Shetzer Y, Solomon H, Koifman G, Molchadsky A, Horesh S, Rotter V. The paradigm of mutant p53-expressing cancer stem cells and drug resistance. Carcinogenesis 2014; 35 : 1196-1208

82. Escoll M, Gargini R, Cuadrado A, Anton IM, Wandosell F. Mutant p53 oncogenic functions in cancer stem cells are regulated by WIP through YAP/TAZ. Oncogene 2017; 36: 3515-3527.

83. Fitamant J, Kottakis F, Benhamouche S, Tian HS, Chuvin N, Parachoniak CA et al. YAP inhibition restores hepatocyte differentiation in advanced $\mathrm{HCC}$, leading to tumor regression. Cell Rep 2015; 10: 1692-1707.

84. Yu F-X, Zhao B, Guan K-L. Regulation of YAP and TAZ transcription co-activators. In: Oren M, Aylon Y(eds) The Hippo Signaling Pathway and Cancer. Springer New York: New York, NY, USA, 2013 pp 71-87

85. Simile MM, Latte G, Demartis MI, Brozzetti S, Calvisi DF, Porcu A et al. Post-translational deregulation of YAP1 is genetically controlled in rat liver cancer and determines the fate and stem-like behavior of the human disease. Oncotarget 2016; 7: 49194-49216.

86. Saladi SV, Ross K, Karaayvaz M, Tata PR, Mou H, Rajagopal J et al. ACTL6A is coamplified with p63 in squamous cell carcinoma to drive YAP activation, regenerative proliferation, and poor prognosis. Cancer Cell 2017; 31: 35-49.

87. Wennmann DO, Vollenbröker B, Eckart AK, Bonse J, Erdmann F, Wolters DA et al. The Hippo pathway is controlled by Angiotensin II signaling and its reactivation induces apoptosis in podocytes. Cell Death Dis 2014; 5: e1519.

88. Tomlinson V, Gudmundsdottir K, Luong P, Leung K-Y, Knebel A, Basu S. JNK phosphorylates Yes-associated protein (YAP) to regulate apoptosis. Cell Death Dis 2010; 1: e29.

89. Fisher ML, Kerr C, Adhikary G, Grun D, Xu W, Keillor JW et al. Transglutaminase interaction with $\alpha 6 / \beta 4$-integrin stimulates YAP1-dependent $\Delta \mathrm{Np} 63 \alpha$ stabilization and leads to enhanced cancer stem cell survival and tumor formation. Cancer Res 2016; 76: 7265-7276.

90. Su X, Napoli M, Abbas HA, Venkatanarayan A, Bui NHB, Coarfa C et al. TAp63 suppresses mammary tumorigenesis through regulation of the Hippo pathway. Oncogene 2016; 36 : 2377-2393

91. Su X, Gi YJ, Chakravarti D, Chan IL, Zhang A, Xia X et al. TAp63 is a master transcriptional regulator of lipid and glucose metabolism. Cell Metab 2012; 16: 511-525.

92. Mohseni M, Sun J, Lau A, Curtis S, Goldsmith J, Fox VL et al. A genetic screen identifies an LKB1-MARK signalling axis controlling the Hippo-YAP pathway. Nat Cell Biol 2014; 16 : 108-117.

93. Colombani J, Polesello C, Josué F, Tapon N. Dmp53 activates the Hippo pathway to promote cell death in response to DNA damage. Curr Biol 2006; 16: 1453-1458.

94. Brodsky MH, Nordstrom W, Tsang G, Kwan E, Rubin GM, Abrams JM. Drosophila p53 binds a damage response element at the reaper locus. Cell 2000; 101: 103-113.

95. Huang J, Wu S, Barrera J, Matthews K, Pan D. The Hippo signaling pathway coordinately regulates cell proliferation and apoptosis by inactivating Yorkie, the Drosophila homolog of YAP. Cell 2005; 122: 421-434.

96. Pantalacci S, Tapon N, Léopold P. The Salvador partner Hippo promotes apoptosis and cell-cycle exit in Drosophila. Nat Cell Biol 2003; 5: 921-927.

97. Yuan $\mathrm{F}$, Xie Q, Wu J, Bai Y, Mao B, Dong $Y$ et al. MST1 promotes apoptosis through regulating Sirt1-dependent p53 deacetylation. J Biol Chem 2011; 286: 6940-6945.

98. Zschoernig B, Mahlknecht U. SIRTUIN 1: regulating the regulator. Biochem Biophys Res Commun 2008; 376: 251-255.

99. Sasaki T, Maier B, Koclega KD, Chruszcz M, Gluba W, Stukenberg PT et al. Phosphorylation regulates SIRT1 function. PLOS ONE 2008; 3: e4020.

100. Guo X, Williams JG, Schug TT, Li X. DYRK1A and DYRK3 promote cell survival through phosphorylation and activation of SIRT1. J Biol Chem 2010; 285: 13223-13232.

101. Hu L, Huang H, Li J, Yin M-X, Lu Y, Wu W et al. Drosophila casein kinase 2 (CK2) promotes warts protein to suppress Yorkie protein activity for growth control. J Biol Chem 2014; 289 : 33598-33607

102. Yang S, Zhang L, Liu M, Chong R, Ding S-J, Chen Y et al. CDK1 phosphorylation of YAP promotes mitotic defects and cell motility and is essential for neoplastic transformation. Cancer Res 2013; 73: 6722-6733.

103. Tschöp K, Conery AR, Litovchick L, Decaprio JA, Settleman J, Harlow E et al. A kinase shRNA screen links LATS2 and the pRB tumor suppressor. Genes Dev 2011; 25: 814-830.

104. Zhou X-H, Yang C-Q, Zhang C-L, Gao Y, Yuan H-B, Wang C. RASSF5 inhibits growth and invasion and induces apoptosis in osteosarcoma cells through activation of MST1/LATS1 signaling. Oncol Rep 2014; 32: 1505-1512.

105. Guo C, Zhang X, Pfeifer GP. The tumor suppressor RASSF1A prevents dephosphorylation of the mammalian STE20-like kinases MST1 and MST2. J Biol Chem 2011; 286: 6253-6261. 
106. Matallanas D, Romano D, Yee K, MeissI K, Kucerova L, Piazzolla D et al. RASSF1A elicits apoptosis through an MST2 pathway directing proapoptotic transcription by the p73 tumor suppressor protein. Mol Cell 2007; 27: 962-975.

107. Song MS, Song SJ, Kim SY, Oh HJ, Lim D-S. The tumour suppressor RASSF1A promotes MDM2 self-ubiquitination by disrupting the MDM2-DAXX-HAUSP complex. EMBO J 2008; 27: $1863-1874$

108. Hamilton G, Yee KS, Scrace S, O'Neill E. ATM regulates a RASSF1A-dependent DNA damage response. Curr Biol 2009; 19: 2020-2025.

109. Basu S, Totty NF, Irwin MS, Sudol M, Downward J. Akt phosphorylates the Yes-associated protein, YAP, to induce interaction with 14-3-3 and attenuation of p73-mediated apoptosis. Mol Cell 2003; 11: 11-23.

110. Strano S, Monti O, Pediconi N, Baccarini A, Fontemaggi G, Lapi E et al. The transcriptional coactivator Yes-associated protein drives p73 gene-target specificity in response to DNA damage. Mol Cell 2005; 18: 447-459.

111. Guo C, Tommasi S, Liu L, Yee J-K, Dammann R, Pfeifer GP. RASSF1A is part of a complex similar to the Drosophila Hippo/Salvador/Lats tumor-suppressor network. Curr Biol 2007; 17: $700-705$

112. Donninger H, Allen N, Henson A, Pogue J, Williams A, Gordon L et al. Salvador protein is a tumor suppressor effector of RASSF1A with hippo pathway-independent functions. J Biol Chem 2011; 286: 18483-18491.

113. Yee KS, Grochola L, Hamilton G, Grawenda A, Bond EE, Taubert H et al. A RASSF1A polymorphism restricts p53/p73 activation and associates with poor survival and accelerated age of onset of soft tissue sarcoma. Cancer Res 2012; 72: 2206-2217.

114. Tian Y, Hou Y, Zhou X, Cheng H, Zhou R. Tumor suppressor RASSF1A promoter: p53 binding and methylation. PLOS ONE 2011; 6: e17017.

115. Zhang H, He J, Li J, Tian D, Gu L, Zhou M. Methylation of RASSF1A gene promoter is regulated by $p 53$ and DAXX. FASEB J 2013; 27: 232-242.

116. Matallanas D, Romano D, Al-Mulla F, O'Neill E, Al-Ali W, Crespo P et al. Mutant K-Ras activation of the proapoptotic MST2 pathway is antagonized by wild-type K-Ras. Mol Cell 2011; 44: 893-906.

117. Aylon Y, Yabuta N, Besserglick H, Buganim Y, Rotter V, Nojima H et al. Silencing of the Lats2 tumor suppressor overrides a p53-dependent oncogenic stress checkpoint and enables mutant H-Ras-driven cell transformation. Oncogene 2009; 28: 4469-4479.

118. Aylon Y, Ofir-Rosenfeld Y, Yabuta N, Lapi E, Nojima H, Lu X et al. The Lats2 tumor suppressor augments p53-mediated apoptosis by promoting the nuclear proapoptotic function of ASPP1. Genes Dev 2010; 24: 2420-2429.

119. Zhang W, Cohen SM. The Hippo pathway acts via p53 and microRNAs to control proliferation and proapoptotic gene expression during tissue growth. Biol Open 2013; 2: 822-828

120. Vigneron AM, Ludwig RL, Vousden KH. Cytoplasmic ASPP1 inhibits apoptosis through the control of YAP. Genes Dev 2010; 24: 2430-2439.

121. Vigneron $\mathrm{AM}$, Vousden $\mathrm{KH}$. An indirect role for ASPP1 in limiting p53-dependent p21 expression and cellular senescence. EMBO J 2012; 31: 471-480.

122. Moroishi T, Park HW, Qin B, Chen Q, Meng Z, Plouffe SW et al. A YAP/TAZ-induced feedback mechanism regulates Hippo pathway homeostasis. Genes Dev 2015; 29 1271-1284.

123. Park G-S, Oh H, Kim M, Kim T, Johnson RL, Irvine KD et al. An evolutionarily conserved negative feedback mechanism in the Hippo pathway reflects functional difference between LATS1 and LATS2. Oncotarget 2016; 7: 24063-24075.

124. Wang C, Zhu X, Feng W, Yu Y, Jeong K, Guo W et al. Verteporfin inhibits YAP function through up-regulating 14-3-3 $\sigma$ sequestering YAP in the cytoplasm. Am J Cancer Res 2016; 6: 27-37

125. Hermeking $\mathrm{H}$, Lengauer $\mathrm{C}$, Polyak K, He TC, Zhang L, Thiagalingam S et al. 14-3-3Sigma is a p53-regulated inhibitor of G2/M progression. Mol Cell 1997; 1: 3-11.

126. Jia Q, Zhou W, Yao W, Yang F, Zhang S, Singh R et al. Downregulation of YAP-dependent Nupr1 promotes tumor-repopulating cell growth in soft matrices. Oncogenesis 2016; 5: e220

127. Xu S, Yang Z, Fan Y, Guan B, Jia J, Gao Y et al. Curcumin enhances temsirolimus-induced apoptosis in human renal carcinoma cells through upregulation of YAP/p53. Oncol Lett 2016; 12: 4999-5006.

128. Bai N, Zhang C, Liang N, Zhang Z, Chang A, Yin J et al. Yes-associated protein (YAP) increases chemosensitivity of hepatocellular carcinoma cells by modulation of $p 53$. Cancer Biol Ther 2013; 14: 511-520.

129. Tebaldi T, Zaccara S, Alessandrini F, Bisio A, Ciribilli Y, Inga A. Whole-genome cartography of p53 response elements ranked on transactivation potential. BMC Genomics 2015; 16: 464.

130. Gao K, An J, Zhang Y, Jin X, Ma J, Peng J et al. The E3 ubiquitin ligase Itch and Yap1 have antagonistic roles in the regulation of ASPP2 protein stability. FEBS Lett 2015; 589 : 94-101.

131. Di Cara F, Maile TM, Parsons BD, Magico A, Basu S, Tapon N et al. The Hippo pathway promotes cell survival in response to chemical stress. Cell Death Differ 2015; 22: 1526-1539.

132. Levy D, Adamovich Y, Reuven N, Shaul Y. The Yes-associated protein 1 stabilizes $p 73$ by preventing Itch-mediated ubiquitination of p73. Cell Death Differ 2007; 14: 743-751.

133. Levy D, Adamovich Y, Reuven N, Shaul Y. Yap1 phosphorylation by c-Abl is a critical step in selective activation of proapoptotic genes in response to DNA damage. Mol Cell 2008; 29: 350-361.
134. Zhang $H$, Wu $S$, Xing $D$. YAP accelerates $A B(25-35)$-induced apoptosis through upregulation of Bax expression by interaction with p73. Apoptosis 2011; 16: 808-821.

135. Van der Weyden L, Papaspyropoulos A, Poulogiannis G, Rust AG, Rashid M, Adams DJ et al. Loss of RASSF1A synergizes with deregulated RUNX2 signaling in tumorigenesis. Cancer Res 2012; 72: 3817-3827.

136. Cottini F, Hideshima T, Xu C, Sattler M, Dori M, Agnelli L et al. Rescue of Hippo coactivator YAP1 triggers DNA damage-induced apoptosis in hematological cancers. Nat Med 2014; 20: 599-606.

137. Ehsanian R, Brown M, Lu H, Yang XP, Pattatheyil A, Yan B et al. YAP dysregulation by phosphorylation or $\Delta$ Np63-mediated gene repression promotes proliferation, survival and migration in head and neck cancer subsets. Oncogene 2010; 29: 6160-6171.

138. Childs BG, Baker DJ, Kirkland JL, Campisi J, van Deursen JM. Senescence and apoptosis: dueling or complementary cell fates? EMBO Rep 2014; 15: 1139-1153.

139. Aylon Y, Oren M. p53: guardian of ploidy. Mol Oncol 2011; 5: 315-323.

140. Rajagopalan H, Lengauer C. Aneuploidy and cancer. Nature 2004; 432: 338-341.

141. Santaguida S, Amon A. Short- and long-term effects of chromosome mis-segregation and aneuploidy. Nat Rev Mol Cell Biol 2015; 16: 473-485.

142. lida S-I, Hirota T, Morisaki T, Marumoto T, Hara T, Kuninaka S et al. Tumor suppressor WARTS ensures genomic integrity by regulating both mitotic progression and G1 tetraploidy checkpoint function. Oncogene 2004; 23: 5266-5274.

143. Ganem NJ, Cornils H, Chiu S-Y, O'Rourke KP, Arnaud J, Yimlamai D et al. Cytokinesis failure triggers hippo tumor suppressor pathway activation. Cell 2014; 158: 833-848.

144. Roy A, Lordier L, Pioche-Durieu C, Souquere S, Roy L, Rameau P et al. Uncoupling of the Hippo and Rho pathways allows megakaryocytes to escape the tetraploid checkpoint. Haematologica 2016; 101: 1469-1478.

145. Schütte U, Bisht S, Heukamp LC, Kebschull M, Florin A, Haarmann J et al. Hippo signaling mediates proliferation, invasiveness, and metastatic potential of clear cell renal cell carcinoma. Transl Oncol 2014; 7: 309-321.

146. Xiao H, Tong R, Yang B, Lv Z, Du C, Peng $C$ et al. TAZ regulates cell proliferation and sensitivity to vitamin D3 in intrahepatic cholangiocarcinoma. Cancer Lett 2016; 381 370-379.

147. Cabochette P, Vega-Lopez G, Bitard J, Parain K, Chemouny R, Masson C et al. YAP controls retinal stem cell DNA replication timing and genomic stability. elife 2015; 4: e08488.

148. Xie $Q$, Chen J, Feng H, Peng S, Adams U, Bai $Y$ et al. YAP/TEAD-mediated transcription controls cellular senescence. Cancer Res 2013; 73: 3615-3624.

149. Kakae K, Ikeuchi M, Kuga T, Saito Y, Yamaguchi N, Nakayama Y. v-Src-induced nuclear localization of YAP is involved in multipolar spindle formation in tetraploid cells. Cell Signal 2017; 30: 19-29.

150. Serrano M, Lin AW, McCurrach ME, Beach D, Lowe SW. Oncogenic ras provokes premature cell senescence associated with accumulation of p53 and p16INK4a. Cell 1997; 88: 593-602.

151. Lin AW, Barradas M, Stone JC, van Aelst L, Serrano M, Lowe SW. Premature senescence involving p53 and p16 is activated in response to constitutive MEK/MAPK mitogenic signaling. Genes Dev 1998; 12: 3008-3019.

152. Fausti F, Di Agostino S, Cioce M, Bielli P, Sette C, Pandolfi PP et al. ATM kinase enables the functional axis of YAP, PML and p53 to ameliorate loss of Werner protein-mediated oncogenic senescence. Cell Death Differ 2013; 20: 1498-1509.

153. Arai $A$, Chano T, Futami $K$, Furuichi $Y$, Ikebuchi $K$, Inui T et al. RECQL1 and WRN proteins are potential therapeutic targets in head and neck squamous cell carcinoma. Cancer Res 2011; 71: 4598-4607.

154. Lee D-H, Park JO, Kim T-S, Kim S-K, Kim T-H, Kim M-C et al. LATS-YAP/TAZ controls lineage specification by regulating TGF $\beta$ signaling and $H n f 4 \alpha$ expression during liver development. Nat Commun 2016; 7: 11961.

155. Yahagi N, Shimano H, Matsuzaka T, Najima Y, Sekiya M, Nakagawa Y et al. p53 Activation in adipocytes of obese mice. J Biol Chem 2003; 278: 25395-25400.

156. Aylon Y, Gershoni A, Rotkopf R, Biton IE, Porat Z, Koh AP et al. The LATS2 tumor suppressor inhibits SREBP and suppresses hepatic cholesterol accumulation. Genes Dev 2016; 30: 786-797.

157. Yahagi N, Shimano H, Matsuzaka T, Sekiya M, Najima Y, Okazaki S et al. p53 involvement in the pathogenesis of fatty liver disease. J Biol Chem 2004; 279: 20571-20575.

158. Sorrentino G, Ruggeri N, Specchia V, Cordenonsi M, Mano M, Dupont S et al. Metabolic control of YAP and TAZ by the mevalonate pathway. Nat Cell Biol 2014; 16: 357-366.

159. Zhang $Y, H u ~ Y$, Fang J-Y, Xu J. Gain-of-function miRNA signature by mutant p53 associates with poor cancer outcome. Oncotarget 2016; 7: 11056-11066.

160. Enzo E, Santinon G, Pocaterra A, Aragona M, Bresolin S, Forcato M et al. Aerobic glycolysis tunes YAP/TAZ transcriptional activity. EMBO J 2015; 34: 1349-1370.

161. DeRan M, Yang J, Shen C-H, Peters EC, Fitamant J, Chan P et al. Energy stress regulates hippo-YAP signaling involving AMPK-mediated regulation of angiomotin-like 1 protein. Cell Rep 2014; 9: 495-503.

162. Mo J-S, Meng Z, Kim YC, Park HW, Hansen CG, Kim S et al. Cellular energy stress induces AMPK-mediated regulation of YAP and the Hippo pathway. Nat Cell Biol 2015; 17 500-510.

163. Wang W, Xiao Z-D, Li X, Aziz KE, Gan B, Johnson RL et al. AMPK modulates Hippo pathway activity to regulate energy homeostasis. Nat Cell Biol 2015; 17: 490-499.

164. Rodriguez OC, Choudhury S, Kolukula V, Vietsch EE, Catania J, Preet A et al. Dietary downregulation of mutant p53 levels via glucose restriction: mechanisms and implications for tumor therapy. Cell Cycle 2012; 11: 4436-4446. 
165. Liang N, Zhang C, Dill P, Panasyuk G, Pion D, Koka V et al. Regulation of YAP by mTOR and autophagy reveals a therapeutic target of tuberous sclerosis complex. J Exp Med 2014; 211: 2249-2263.

166. Wilkinson DS, Jariwala JS, Anderson E, Mitra K, Meisenhelder J, Chang JT et al. Phosphorylation of LC3 by the Hippo kinases STK3/STK4 is essential for autophagy. Mol Cell 2015; 57: 55-68.

167. Nishioka N, Inoue K, Adachi K, Kiyonari H, Ota M, Ralston A et al. The Hippo signaling pathway components Lats and Yap pattern Tead4 activity to distinguish mouse trophectoderm from inner cell mass. Dev Cell 2009; 16: 398-410.

168. Fallahi E, O'Driscoll NA, Matallanas D. The MST/Hippo pathway and cell death: a noncanonical affair. Genes (Basel) 2016; 7.

169. Furth N, Bossel Ben-Moshe N, Pozniak Y, Porat Z, Geiger T, Domany E et al. Downregulation of LATS kinases alters p53 to promote cell migration. Genes Dev 2015; 29 : 2325-2330.

170. Kandoth C, McLellan MD, Vandin F, Ye K, Niu B, Lu C et al. Mutational landscape and significance across 12 major cancer types. Nature 2013; 502: 333-339.

171. Tong Q, Mazur SJ, Rincon-Arano H, Rothbart SB, Kuznetsov DM, Cui G et al. An acetylmethyl switch drives a conformational change in p53. Structure 2015; 23: 322-331.

172. Emamzadah S, Tropia L, Vincenti I, Falquet B, Halazonetis TD. Reversal of the DNAbinding-induced loop L1 conformational switch in an engineered human p53 protein. $J \mathrm{Mol}$ Biol 2014; 426: 936-944.

173. Rivlin N, Katz S, Doody M, Sheffer M, Horesh S, Molchadsky A et al. Rescue of embryonic stem cells from cellular transformation by proteomic stabilization of mutant p53 and conversion into WT conformation. Proc Natl Acad Sci USA 2014; 111 7006-7011.

174. Trinidad AG, Muller PAJ, Cuellar J, Klejnot M, Nobis M, Valpuesta JM et al. Interaction of p53 with the CCT complex promotes protein folding and wild-type p53 activity. Mol Cell 2013; 50: 805-817.

175. Gogna R, Madan E, Kuppusamy P, Pati U. Re-oxygenation causes hypoxic tumor regression through restoration of p53 wild-type conformation and post-translational modifications. Cell Death Dis 2012; 3: e286.

176. Zhang W, Deisseroth $\mathrm{AB}$. Conformational change of $\mathrm{p} 53$ protein in growth factor-stimulated human myelogenous leukemia cells. Leuk Lymphoma 1994; 14: 251-255.
177. Moroishi T, Hansen CG, Guan K-L. The emerging roles of YAP and TAZ in cancer. Nat Rev Cancer 2015; 15: 73-79.

178. Ferraiuolo M, Verduci L, Blandino G, Strano S. Mutant p53 protein and the hippo transducers YAP and TAZ: A critical oncogenic node in human cancers. Int $\mathrm{J} \mathrm{Mol} \mathrm{Sci}$ 2017; 18.

179. Labi V, Erlacher M. How cell death shapes cancer. Cell Death Dis 2015; 6: e1675.

180. Luke JJ, Van De Wetering $\mathrm{Cl}$, Knudson CM. Lymphoma development in Bax transgenic mice is inhibited by Bcl-2 and associated with chromosomal instability. Cell Death Differ 2003; 10: 740-748.

181. Kruiswijk F, Labuschagne CF, Vousden KH. p53 in survival, death and metabolic health: a lifeguard with a licence to kill. Nat Rev Mol Cell Biol 2015; 16: 393-405.

182. Gao Y, Zhang W, Han X, Li F, Wang X, Wang R et al. YAP inhibits squamous transdifferentiation of Lkb1-deficient lung adenocarcinoma through ZEB2-dependent DNp63 repression. Nat Commun 2014; 5: 4629.

183. Lapi E, Di Agostino S, Donzelli S, Gal H, Domany E, Rechavi G et al. PML, YAP, and p73 are components of a proapoptotic autoregulatory feedback loop. Mol Cell 2008; 32: 803-814.

184. Sugawara W, Arai $Y$, Kasai F, Fujiwara $Y$, Haruta M, Hosaka R et al. Association of germline or somatic TP53 missense mutation with oncogene amplification in tumors developed in patients with Li-Fraumeni or Li-Fraumeni-like syndrome. Genes Chromosomes Cancer 2011; 50: 535-545.

185. Zender L, Spector MS, Xue W, Flemming P, Cordon-Cardo C, Silke J et al. Identification and validation of oncogenes in liver cancer using an integrative oncogenomic approach. Cell 2006; 125: 1253-1267.

186. Eisinger-Mathason TSK, Mucaj V, Biju KM, Nakazawa MS, Gohil M, Cash TP et al. Deregulation of the Hippo pathway in soft-tissue sarcoma promotes FOXM1 expression and tumorigenesis. Proc Natl Acad Sci USA 2015; 112: E3402-E3411.

187. Pandit B, Halasi M, Gartel AL. p53 negatively regulates expression of FoxM1. Cell Cycle 2009; 8: 3425-3427.

188. Weiler SME, Pinna F, Wolf T, Lutz T, Geldiyev A, Sticht $C$ et al. Induction of chromosome instability by activation of yes associated protein and forkhead box M1 in liver cancer. Gastroenterology 2017; 152: 2037-2051.e22. 